Elisabeth Vocks ${ }^{1,2}$

S. Borelli ${ }^{2,3}$

R. Busch 4

H. Düngemann 5 J. Ring ${ }^{1,2}$

\section{Biometrische Studie zur Wetterabhängigkeit und Wetterempfindlichkeit beim atopischen Ekzem}

Biometric Study on Weather Dependence and Weather Sensibility

in Atopic Eczema

\section{Zusammenfassung}

Die Abhängigkeit des atopischen Ekzems von meteorologischen Bedingungen wurde mit Hilfe von Fragebogen zur Juckreizstärke, Wetterempfinden sowie meteorologischen Messungen bei insgesamt 2106 Patienten mit atopischem Ekzem in Davos, Schweizer Hochgebirge, untersucht. Die Beobachtungsperiode betrug 7 Jahre. Es konnten hochsignifikante Korrelationen zwischen der Pruritusstärke und der Ausprägung meteorologischer Einzelparameter, insbesondere eine negative Beziehung zwischen der Stärke des Pruritus und der Höhe der Lufttemperatur, des Luftdampfdrucks und der Sonnenscheindauer ermittelt werden, darüber hinaus erhöhte subjektive Empfindlichkeitsraten der Patienten für Luftfeuchtigkeit und Lufttrockenheit. Damit wurden erstmals Daten erhoben, die die besondere Relevanz der atmosphärischen Umwelt für die Symptomausprägung beim atopischen Ekzem belegen. Die Ergebnisse lassen vor allem darauf schließen, dass ein mittlerer thermisch-hygrischer Bereich, bei dem sich der Wärmeübergang an der Hautoberfläche und der epidermale Wassergehalt die Balance halten, therapeutisch günstige Einflüsse besitzt. Die eingeschränkte klimatische Toleranz ist Folge der gestörten kutanen Barriere beim atopischen Ekzem.

\section{Abstract}

The dependence of atopic eczema on meteorological conditions was investigated by questionnaire about itch intensity and subjective weather sensations, compared to meteorological measurement data. The study, which was carried out in the high mountain valley of Davos, Switzerland, included a total of 2,106 atopic eczema patients and comprised an observation period of 7 years. Highly significant correlations between itch and meteorological parameter intensity were found, especially an inverse correlation between itch intensity and air temperature, vapor pressure and sunshine duration could be ascertained, combined with an increased subjective sensibility for air humidity and dryness. By these data the particular influence of atmospheric environment on symptom intensity in atopic eczema is verified for the first time. It can be concluded that a medium thermo-hygric meteorological range providing a balance between heat and water loss of the skin surface is most favourable for atopic eczema patients. The limited meteorological tolerance is due to the disturbed cutaneous barrier function in atopic eczema.

\section{Einleitung}

Das atopische Ekzem ist eine häufige Hautkrankheit mit einer geschätzten Prävalenz von ca. 10\%. Der klinische Verlauf ist wechselhaft und sehr individuell schwankend. Bezüglich klimatischer Faktoren werden häufig Verschlechterungen der ekzema- tösen Hautveränderungen während der kalten Jahreszeiten angegeben [1,2]. Ebenso können Wetterwechsel Juckreizverstärkung auslösen [1]. In wärmeren Ländern soll die Prävalenz des atopischen Ekzems nach Meinung einiger Autoren niedriger sein [3 -5], jedoch suchen Neurodermitiker weltweit therapeutische Klimawirkungen doch in eher kühleren Klimaregionen [5];

${ }^{1}$ Klinik und Poliklinik für Dermatologie und Allergologie am Biederstein der Technischen Universität München

${ }^{2}$ Klinik für Dermatologie und Allergologie - Alexanderhausklinik - Davos (Schweiz) ${ }^{3}$ Forschungsgruppe Noxenkatalog am Biederstein, München ${ }^{4}$ Institut für medizinische Statistik und Epidemiologie der Technischen Universität München ${ }^{5}$ Effnerstraße 73, München 
Klimatherapien der Neurodermitis an der Nordsee oder im Hochgebirge haben sich diesbezüglich als besonders effektiv erwiesen [6-8].

Ein Zusammenhang des Neurodermitisverlaufes mit dem umgebenden Klima ist plausibel, da die Haut bei Neurodermitis neben immunologischen $[4,9]$ auch nichtimmunologische konstitutionelle Besonderheiten aufweist, vor allem eine abnorme kutane Gefäßregulation $[10,11]$ und eine gestörte Barrierefunktion des Stratum corneum $[12,13]$. Die fehlende funktionelle und morphologische Integrität der Haut beim atopischen Ekzem führt möglicherweise schneller zu Adaptationsstörungen und Irritationen [14] bei Veränderung der meteorologischen Umgebungsfaktoren.

Angesichts der steigenden Prävalenz $[15,16]$ dieser letztlich ätiologisch nicht vollständig geklärten nur schwer zu behandelnden Dermatose $[9,15,17]$ kommt auch der Frage nach besserer Charakterisierung nichtimmunologischer Störungen, insbesondere der Abhängigkeit des atopischen Ekzems vom Umgebungsklima, zunehmende Bedeutung zu.

In dieser Untersuchung wurden die Beziehungen zwischen veränderlichen Wetterelementen und dem Neurodermitisverlauf einerseits und dem subjektiven Wetterempfinden andererseits analysiert. Es sollte festgestellt werden, ob unter natürlichen Bedingungen signifikante Zusammenhänge zwischen Wetter und Krankheitssymptomen nachweisbar sind und inwieweit sich mögliche Abweichungen in der meteorologischen Befindlichkeit auf den Neurodermitisverlauf auswirken.

\section{Material und Methoden}

\section{Patienten}

2106 stationäre erwachsene Patienten (Ø-Alter 33,5 Jahre, $\mathrm{m}: \mathrm{w}=1: 1,7)$ der Deutschen Klinik für Dermatologie und Allergie - Alexanderhausklinik - im Schweizer Hochtal von Davos/ Graubünden, $1560 \mathrm{~m}$ ü. NN, mit der Diagnose Neurodermitis constitutionalis atopica/syn. atopisches Ekzem wurden untersucht. Die durchschnittliche Aufenthaltsdauer der Patienten in Davos betrug 34,1 Tage, der Gesamtzeitraum der Beobachtung 7 Jahre.

Die Schweregrade der Neurodermitis, klassifiziert in Anlehnung an den „atopic eczema area and severity index“ (AEASI) [18], rangierten von leichten bis schweren Neurodermitisfällen. Die Therapie bestand in klassischer Dermatotherapie unter nur geringer und im Therapieverlauf abnehmender Verwendung von topischen Kortikosteroiden und Hautpflegemaßnahmen sowie Heliotherapie.

Pruritus: Zur Verlaufsbeurteilung der Neurodermitis wurde das klinische Leitsymptom Pruritus gewählt. Die Angaben über die Juckreizstärke erfolgten täglich (nachmittags) in vier Stufen (Ordinalskala): (kein Juckreiz $=0$, leichter Juckreiz $=1$, mittlerer Juckreiz $=2$, starker Juckreiz $=3$ ).

Die Bewertung erfolgte als arithmetisches Mittel der Juckreizstärken aller Patienten eines Tages ( $=$ mittlerer täglicher Pruritus, syn. Pruritustagesmittel[wert]).
Subjektives Wetterempfinden: Die Patienten gaben täglich (nachmittags) an, ob sie das Wetter am betreffenden Tag als trocken, feucht und/oder windig empfanden (ja/keine Angabe). Die täglichen Angaben wurden bezüglich jeder einzelnen Qualität (trocken, feucht, windig) für die Patienten, die diese Angabe bejahten, in Prozent aller Patienten, die an diesem Tag an der Untersuchung beteiligt waren, berechnet.

\section{Klima- und Wetterdaten}

Die Klima- und Wetterdaten wurden durch die Schweizerische Meteorologische Anstalt (SMA) im Physikalisch-Meteorologischen Observatorium Davos/Weltstrahlungszentrum (PMOD/ WRC) täglich gemessen. Die Messungen sind entsprechend dem „Commission for Instruments and Methods of Observation (CIMO)-Guide“ genormt.

Die Datenerfassung erfolgte gemäß WMO (World Meteorological Organisation)-Spezifikation.

1. Lufttemperatur ( $\operatorname{Grad}$ Celsius $\left.\left[{ }^{\circ} \mathrm{C}\right]\right)$

2. Luftdruck (Hektopascal [hPa])

3. Relative Luftfeuchtigkeit (Prozent [\%])

4. Dampfdruck (Hektopascal [hPa])

5. Mittlere Windgeschwindigkeit (Knoten [kn])

6. Bewölkungsmittel (1/8 vom Himmel)

7. Sonnenscheindauer (Tagessumme in Stunden [h])

(bei 1. -6. jeweils das arithmetische Mittel aller Stundenwerte eines Tages für die Messzeiten 7, 13 und 19 Uhr Ortszeit).

\section{Auswertung}

Die Datenauswertung erfolgte mit dem Statistikprogramm „Statistisches Paket für Soziale Statistik“" (SPSS, Version 3.0) auf dem Rechnersystem MX300 SINIX-V. 5.21.

Neben Regressionsanalysen für kontinuierliche Merkmale (univariat bzw. multipel) kamen der Spearman-Korrelationskoeffizient für nicht kontinuierliche Merkmale, der Mann-WhitneyRangsummen-Test (Vergleich von zwei Gruppen) und der Kruskal-Wallis-Test (Vergleich von mehr als zwei Gruppen) zur Anwendung.

Da bei der Fragestellung und dem vorliegenden Datenmaterial Hypothesen generiert werden sollten, wurde die Auswertung im Sinne einer explorativen Datenanalyse durchgeführt. Das Signifikanzniveau wurde auf $5 \%(p<0,05)$ festgelegt.

Für die Korrelationsuntersuchungen zwischen klinischen und meteorologischen Parametern wurden nur Tage, an denen mindestens 20 Patienten beteiligt waren, in die Auswertungen einbezogen.

\section{Ergebnisse}

Von 2557 Kalendertagen (1983-1989) waren insgesamt 1623 Tage mit jeweils mindestens 20 Studienpatienten (Mittelwert 33,2 Pat.) auswertbar. Die Fallzahlen pro Tag waren bezüglich Schweregrad und Aufenthaltsdauer ausgeglichen. 


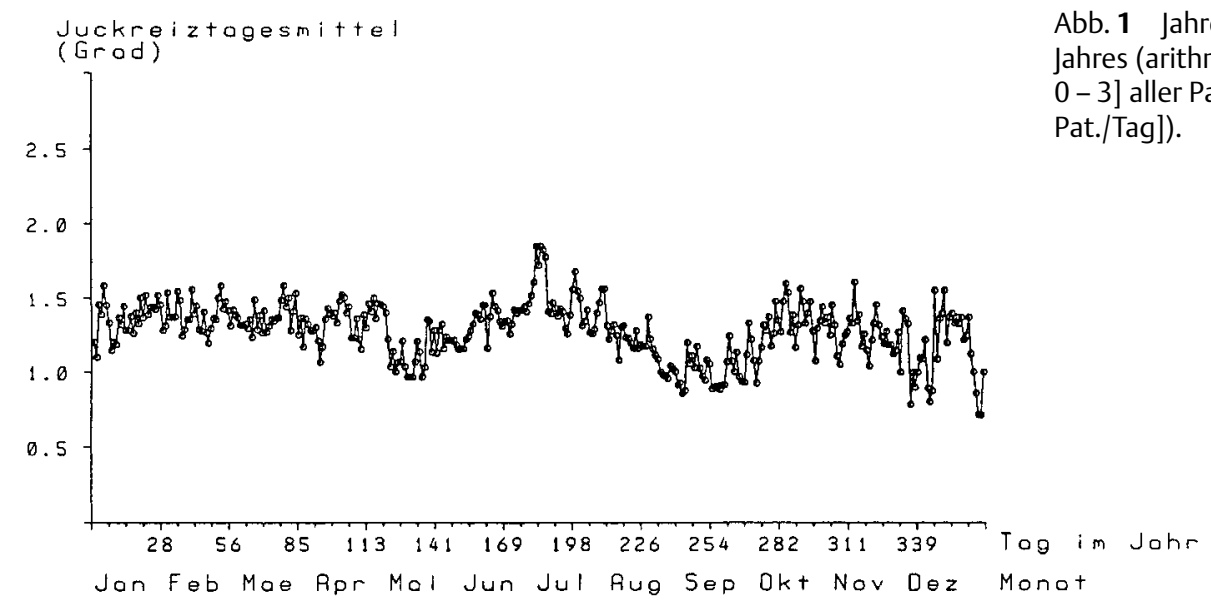

Abb. 1 Jahresverlauf der Pruritustagesmittel, Beispiel eines ahres (arithmetisches Mittel der Pruritusstärken [Skala von 0 - 3] aller Patienten eines Tages [durchschnittlich 33,2 Pat./Tag])

\section{Pruritus}

Das durchschnittliche Pruritustagesmittel aller Patienten lag im Median (Med.) bei 1,220 (Mittelwert 1,224/Standardabweichung 0,180/Minimum 0,700/Maximum 1,850).

Bezüglich der mittleren täglichen Juckreizstärke bestand kein Unterschied zwischen den Geschlechtern, Altersgruppen, ebenso nicht zwischen den Neurodermitikern ohne (32\% [664/2106]) und mit (68\% [1442/2106]) atopischen Schleimhauterkrankungen. Ein hochsignifikanter Unterschied im Juckreiztagesmittel bestand zwischen Patienten in der ersten Aufenthaltshälfte (Med. 1,320) und denen in der zweiten Aufenthaltshälfte (Med. $1,110)(p=0,0000)$ der stationären Therapie, was die klinische Besserung im Verlauf der Therapie widerspiegelt.

Der Verlauf des mittleren täglichen Pruritus der Patienten ergibt wellenförmige Kurven mit vergleichsweise geringen Tagesamplituden, aber einer deutlichen Bewegung im mittleren Kurvenverlauf (Abb.1). Daraus geht hervor, dass ein Kollektiv von mindestens 20 Patienten/Tag groß genug ist, um individuelle Einflussfaktoren auf den täglichen Pruritus der Gruppe (z.B. unterschiedliche Schweregrade, Therapien, Therapiestadien, psychische Faktoren, mögliche Allergenexposition etc.) auszugleichen und die Beurteilung kollektiver Einflussfaktoren zu erlauben. Der Pruritus erwies sich somit als ein valider Messparameter.

\section{Subjektives Wetterempfinden}

Im Durchschnitt empfanden im Gesamtzeitraum 60\% aller Patienten das Wetter als trocken, $22 \%$ als feucht und $9 \%$ als windig, als indifferent nur $9 \%$. Die Wetterempfindungsqualitäten korrelierten miteinander wie folgt: „trocken“ - „feucht“: - 0,741; „trocken“- „windig“: - 0,247; „feucht“- „windig“: + 0,283 (jeweils Korrelationskoeffizient [Korr.koeff.], $p<0,001$ ) und gut mit objektiven Klimaparametern, besonders die Angaben zu „trocken“/,feucht“" mit der gemessenen relativen Luftfeuchtigkeit und „windig“ mit der gemessenen Windgeschwindigkeit (Tab.1). Die Korrelationen sprechen einerseits für eine gute Diskriminierungsfähigkeit der Patienten bezüglich thermisch-hygrischer Umgebungsbedingungen (trocken vs. feucht) und damit für eine hohe Validität dieser subjektiven Parameter. Andererseits zeigen sie auf, dass Luftbewegung umso stärker wahrgenommen wird, je höher die Luftfeuchtigkeit ist; diesbezüglich unterscheiden sich die untersuchten Neurodermitiker meteorotropisch nicht von Hautgesunden $[19,20]$.
Tab. 1 Korrelationen des subjektiven Wetterempfindens mit objektiven meteorologischen Parametern (Gesamtzeitraum)

\begin{tabular}{|c|c|c|c|}
\hline \multirow[t]{2}{*}{ meterologische Parameter } & \multicolumn{3}{|c|}{ subjektives Wetterempfinden } \\
\hline & „trocken“ & "feucht“ & „windig“ \\
\hline Lufttemperatur & & $-0,117$ & \\
\hline relative Luftfeuchtigkeit & $-0,624$ & $+0,657$ & \\
\hline Dampfdruck (absolute Luftfeuchtigkeit) & & $+0,162$ & \\
\hline Windgeschwindigkeit & $-0,277$ & $+0,311$ & $+0,473$ \\
\hline
\end{tabular}

jeweils Korrelationskoeffizienten (0,0: kein Zusammenhang, $\pm 1,0: 100 \%$ iger positiver/negativer Zusammenhang) (Irrtumswahrscheinlichkeit $\mathrm{p}<0,001$ )

\section{Beziehungen zwischen Pruritus und meteorologischen Parametern \\ Univariate und multiple Regression - Gesamtzeitraum}

Die univariaten Regressionsanalysen zwischen Juckreiztagesmittelwerten und den Tagesmittelwerten der einzelnen Klimaparameter sind in Tab. 2 aufgeführt. Die Zusammenhänge mit Pruritus waren fast durchweg hochsignifikant. Demnach nimmt der Pruritus mit Zunahme von Lufttemperatur, Luftdruck, Dampfdruck, Windgeschwindigkeit und Sonnenscheindauer signifikant ab und mit Zunahme von Bewölkung signifikant zu (Tab. 2). Die Einzelkorrelationen sind dabei nicht sehr eng, der höchste absolute Korrelationskoeffizient ist 0,235 für die inverse Beziehung zwischen Pruritusstärke und Lufttemperatur.

Mittels multipler Regression konnte lediglich die Korrelation zwischen Pruritus und Lufttemperatur durch Kombination mit Sonnenscheindauer noch gering verbessert werden (Korr.koeff. absolut $0,242, p<0,001)$. Engere Korrelationen wurden hiermit nicht verifiziert.

\section{Univariate Regression - Zeitabschnitte}

Wenn die klimatischen Variablen einen signifikanten Einfluss auf die Pruritusstärke haben, kann erwartet werden, dass dieser am deutlichsten zutage tritt, wenn sich das Wetter stärker ändert. Deshalb wurden aus den Jahreskurven der einzelnen meteorologischen Parameter Abschnitte herausgegriffen, in denen sich die Parameter stärker änderten, und in diesen Zeitperioden univariate Regressionsanalysen zwischen meteorologischen Parametern und der Pruritusstärke durchgeführt. Ein Beispiel zu so einer „Fenster“-Analyse ist in der Abb. 2 zu sehen. In solchen 
Tab. 2 Korrelationen der Pruritustagesmittel mit den verschiedenen Klimaeinzelparametern - univariate Regressionen - Gesamtzeitraum und Zeitabschnitte (,Fenster“)

\begin{tabular}{|c|c|c|c|c|}
\hline \multirow[t]{3}{*}{ meteorologische Parameter } & \multicolumn{4}{|c|}{ Korrelationen mit Pruritusstärke } \\
\hline & \multicolumn{2}{|c|}{$\begin{array}{c}\text { Gesamtzeitraum } \\
(1623 \text { Tage })\end{array}$} & \multicolumn{2}{|c|}{$\begin{array}{c}\text { Zeitabschnitte (,Fenster“) } \\
\text { (min. 16, max. } 50 \text { Tage) }\end{array}$} \\
\hline & $\begin{array}{l}\text { Korrelationskoeffizient } \\
r\end{array}$ & Irrtumswahrscheinlichkeit & $\begin{array}{l}\text { Korrelationskoeffizienten } \\
r\end{array}$ & $\begin{array}{l}\text { Irrtumswahrscheinlichkeit } \\
\text { p }\end{array}$ \\
\hline Lufttemperatur & $-0,235$ & $<0,001$ & $-0,573 /-0,587$ & $<0,001$ \\
\hline Luftdruck & $-0,124$ & $<0,001$ & $-0,79 /-0,579 /-0,468$ & $\leq 0,001$ \\
\hline relative Luftfeuchtigkeit & $+0,044$ & n.s. & $+0,062 /+0,307$ & n.s. \\
\hline Dampfdruck & $-0,180$ & $<0,001$ & $-0,605 /-0,640$ & $<0,001$ \\
\hline Windgeschwindigkeit & $-0,064$ & 0,005 & $-0,067$ & n.s. \\
\hline Sonnenscheindauer & $-0,120$ & $<0,001$ & $-0,587$ & 0,013 \\
\hline Bewölkung & $-0,077$ & $<0,001$ & nichtlinear* & 0,042 \\
\hline
\end{tabular}

Korrelationskoeffizient r 0,0: kein Zusammenhang, \pm 1,0:100\%iger positiver/negativer Zusammenhang; * niedrigste Pruritusmittelwerte (Mean Rank) bei mittlerer Bewölkung (2/8 oder 4/8) und höhere bei klarem (0/8) und bei $100 \%$ bedecktem (8/8) Himmel; n. s.: nicht signifikant

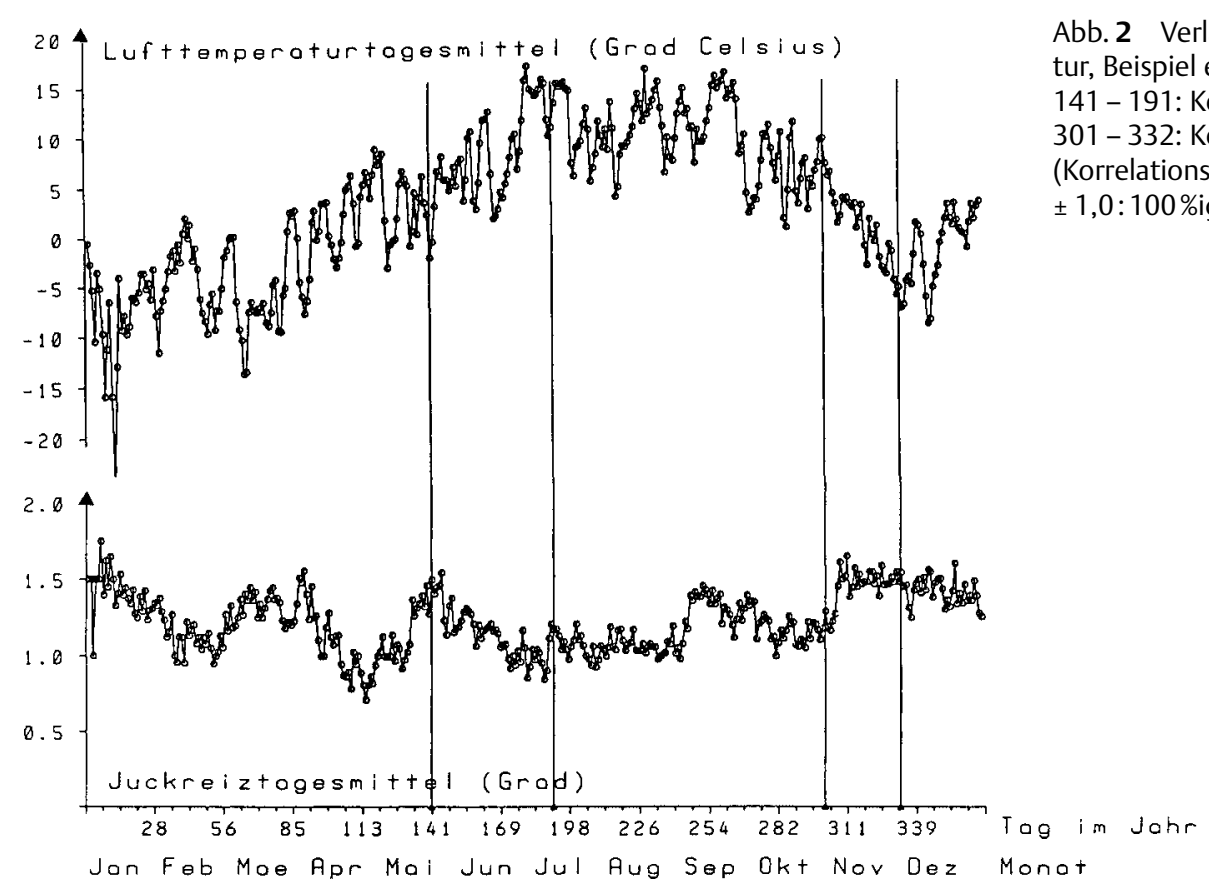

ausgewählten Zeitabschnitten ergeben sich deutlich engere Korrelationen (Korr.koeff. absolut $>0,6, p<0,001$ ). Geprüft für alle Parameter ließen sich für Lufttemperatur, Luftdruck, Dampfdruck und Sonnenscheindauer enge Korrelationen nachweisen (Tab. 2). Mit dieser „Fenster“-Methode konnte gezeigt werden, dass es Perioden gibt, in denen die Korrelationen wesentlich deutlicher zutage treten.

\section{Pruritusunterschiede bei verschiedenen meteorologischen Konstellationen}

Um die sich offensichtlich überlagernden Effekte von relativer Luftfeuchtigkeit und Windgeschwindigkeit zu differenzieren, wurden verschiedene Klimamerkmale kombiniert, und der Juckreiz an den Tagen, die diese Merkmale aufwiesen, mit dem Juckreiz an jeweils allen anderen Tagen verglichen (Tab. 3). Als Grenze zwischen höheren oder niedrigeren Bereichen der einzelnen meteorologischen Parameter galt jeweils der Mittelwert des Gesamtzeitraumes (s. a. Tab. 4, rechte Spalte).
Der Pruritus war an wärmeren Tagen $\left(>3,1^{\circ} \mathrm{C}\right)$ signifikant geringer und an kälteren Tagen $\left(<3,1^{\circ} \mathrm{C}\right)$ signifikant stärker als an jeweils allen übrigen Tagen. Höhere Luftfeuchtigkeit (> 73\%) bewirkte sowohl an warmen als auch an kalten Tagen einen stärkeren Pruritus, der Unterschied war aber nicht signifikant (Tab. 3).

Unterschiedliche Windgeschwindigkeiten bewirkten an allen wärmeren und an kalt-trockenen Tagen keine Pruritusunterschiede, an kalt-feuchten Tagen aber waren höhere Windgeschwindigkeiten mit einer signifikanten Pruritusreduktion verbunden. Unter den gewählten 3-Parameter-Bedingungen ergab sich an kalten-windarmen Tagen auch ein signifikanter Pruritusunterschied in Abhängigkeit von der relativen Luftfeuchtigkeit mit signifikanter Prurituszunahme bei höherer relativer Luftfeuchtigkeit (Tab. 3). 
Beziehungen zwischen subjektivem Wetterempfinden, meteorologischen Parametern und Pruritus

Die genaueren Beziehungen zwischen subjektivem Wetterempfinden und objektiven Klimaparametern zeigt Tab.4. Mehr als $50 \%$ aller Patienten empfanden das Wetter schon als feucht, wenn die relative Luftfeuchtigkeit durchschnittlich $86 \%$, also nicht sehr hoch, die Luftemperatur $2,9^{\circ} \mathrm{C}$ und die Windgeschwindigkeit 6,4 Knoten waren, und bereits als trocken, wenn Bedingungen von durchschnittlich noch $67 \%$ relative Luftfeuchtigkeit, $5,1^{\circ} \mathrm{C}$ Lufttemperatur und $4,7 \mathrm{kn}$ Windgeschwindigkeit vorherrschten (Tab. 4).

Zwischen subjektiver Wetterempfindungsqualität und Pruritusstärke konnte trotz niedriger Irrtumswahrscheinlichkeit keine nennenswerte Korrelation nachgewiesen werden (Korr.koeff. zwischen Pruritusstärke und „Wetterempfinden trocken“: - 0,081, „Wetterempfinden feucht“: + 0,071, „Wetterempfinden windig“: + 0,065; $[p \leq 0,009])$.
Diskussion

Die anhand der Analyse von über 2000 Patienten und meteorologischen Daten von 7 Jahren gewonnenen Ergebnisse zeigen signifikante Beziehungen zwischen veränderlichen meteorologischen Bedingungen und der Juckreizintensität beim atopischen Ekzem. Darüber hinaus wurde anhand des subjektiven Wetterempfindens eine eingeschränkte Toleranzbreite bezüglich thermisch-hygrischer Umgebungsbedingungen ermittelt. Damit werden die bisher nur empirischen Hinweise $[1,5,16]$ bestätigt, dass das umgebende Klima für den Verlauf der Neurodermitis, einer Erkrankung mit konstitutioneller Störung der kutanen Barrierefunktion, ein äußerst relevanter Kofaktor ist.

Als repräsentativer Parameter für den Neurodermitisverlauf wurde in dieser bioklimatologischen Untersuchung der Pruritus gewählt. Der Juckreiz ist beim atopischen Ekzem ein Kardinalsymptom und eng mit dem klinischen Verlauf verknüpft $[1,4,15]$. Er wird zwar subjektiv empfunden und ist nicht objektiv messbar [21,22], wobei seine Stärke individuell unterschiedlich, meist in Relation zum eigenen mittleren Pruritus beurteilt wird [22]; der Pruritus ist aber gerade deshalb für die vorliegen-

Tab. 3 Pruritustagesmittel an Tagen mit jeweils verschiedenen Temperatur-, Luftfeuchtigkeits- und Windbedingungen (Gesamtzeitraum)

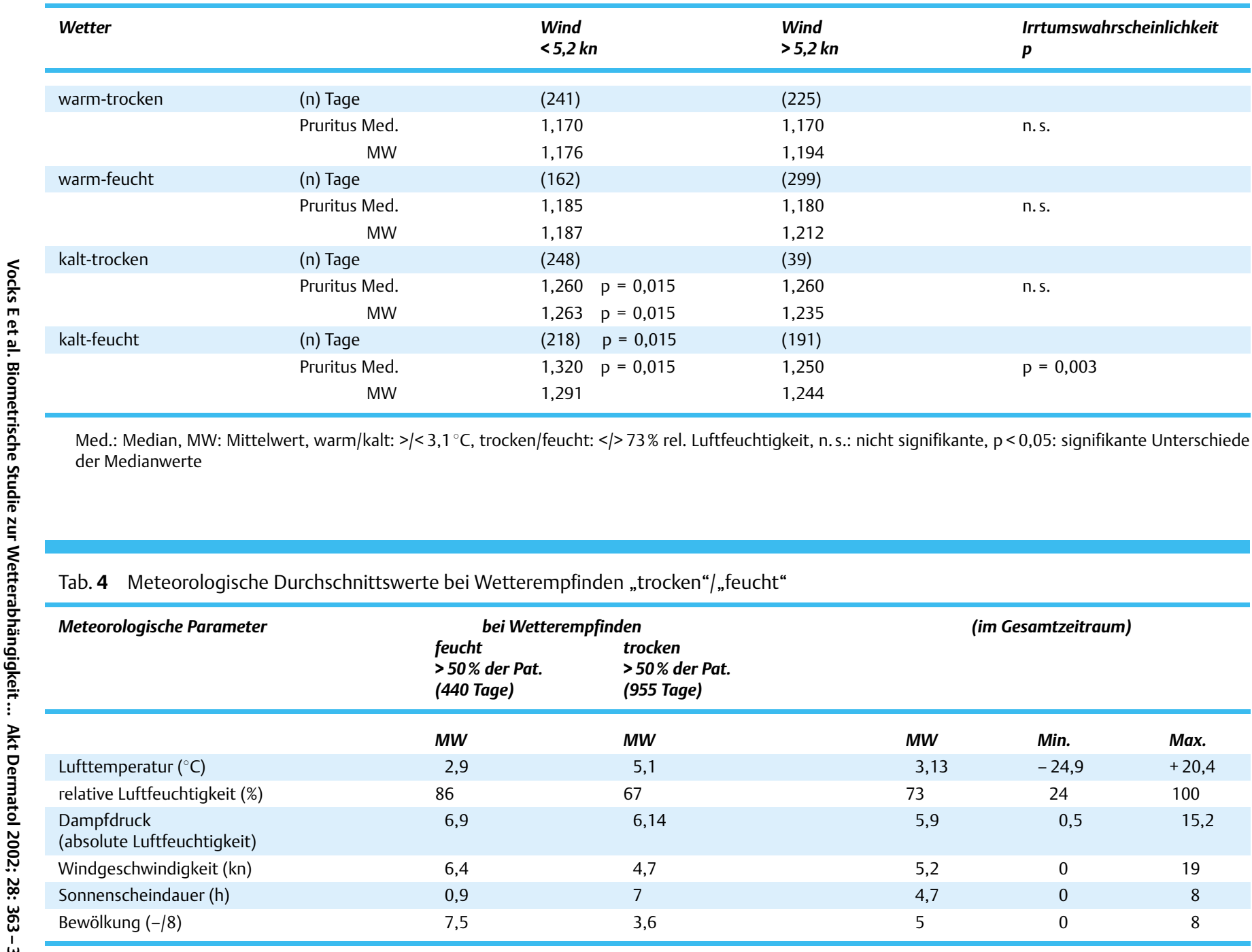

MW: Mittelwert, Min.: Minimum, Max.: Maximum (Tagesmittel) 
de Untersuchung ein besonders geeigneter Parameter, da die Veränderung des Krankheitsverlaufes in Abhängigkeit von meteorologischen Bedingungen untersucht werden sollte, und damit eher die relative als die absolute Symptomstärke von Interesse war. Da der Juckreiz früh reagiert, oft vor Auftreten von Hauterscheinungen [15], ist er gleichzeitig der empfindlichste Parameter.

Zur Erfassung der klimatischen Einflüsse wurden meteorologische Einzelmessgrößen herangezogen, wenngleich die Parameter nie unabhängig voneinander wirken. In Formeln definierte meteorologische Komplexgrößen, wie „Abkühlgröße“, „Effektivtemperatur", um nur einige zu nennen $[19,20,23]$, sind in der modernen human-biometeorologischen Forschung zunehmend wieder verlassen worden, da sie letztendlich künstlichen Modellen entsprechen und deshalb potenziell zu Fehlschlüssen führen können.

Zwischen Juckreizstärke und den meisten meteorologischen Parametern ergaben sich hochsignifikante Korrelationen. Solche Signifikanzen können sich bei biometeorologischen „Feld“untersuchungen erst ab einem großen Datenpool zeigen und setzen somit eine immense Datenfülle und moderne Methoden der Datenverarbeitung voraus; dadurch erklärt sich auch, dass ähnliche Untersuchungen in früheren Jahren ohne Ergebnis blieben. Die ermittelten Korrelationen mit absoluten Koeffizienten um 0,1-0,24 sind, gemessen an vergleichbaren human-biometeorologischen Untersuchungen, als valide zu bewerten; wirklich enge Zusammenhänge (Korr.koeff. absolut $\geq 0,6$ ) zwischen atmosphärischen Einzelparametern und untersuchten Bedingungen am Menschen können sich infolge der Multivariabilität eines Klimas naturgemäß praktisch nie ergeben [20,24]. Wie unsere Untersuchung auch zeigte, sind multiple Regressionsanalysen hier methodisch nicht wesentlich überlegen. Wesentlich engere Korrelationen zwischen meteorologischen Parametern und der Pruritusstärke konnten hingegen in gezielt ausgesuchten Zeitabschnitten, in denen sich der jeweils untersuchte Parameter stärker veränderte, aufgezeigt werden. Diese statistisch zulässigen „Fenster“-Analysen dienen zur Bestätigung von Trends. Stärker überlagerte Zusammenhänge bedurften weiterer Aufschlüsselungen; insbesondere die Auswirkungen von höherer relativer Luftfeuchtigkeit und Windgeschwindigkeit waren unter den Davoser Bedingungen nur schwer zu erfassen, weil sie im Klima der geschützten Hochgebirgstallage praktisch nicht vorkommen.

Erstaunlicherweise stellte sich die Lufttemperatur als der relevanteste Parameter heraus. Unter den eher kalten Bedingungen des Hochgebirges (s. a. Tab. 4, rechte Spalte) nahm der Pruritus mit zunehmender Lufttemperatur, bis ca. $18^{\circ} \mathrm{C}$ (durchschnittliches Maximum im Sommer), ab. Das wesentliche Moment ist hier zum einen in der temperaturabhängigen Bekleidung zu sehen: je höher die Lufttemperaturen, umso luftdurchlässiger (im Vergleich zum Winter) ist die Kleidung und umso größer ist die Wärmeabgabe an der Hautoberfläche mittels Abstrahlung, Konvektion und Verdunstung [19,20,23]. Förderlich sind weiterhin eine mittlere Luftbewegung und eine für Außenbedingungen niedrigere relative Luftfeuchtigkeit $<73 \%$, wie unsere Daten ebenfalls zeigen. Letztere erleichtert den transepidermalen Wasserverlust (TEWL) [25] und damit die Bildung von Verdunstungskälte. Hautoberflächenkühlung wirkt antiinflammatorisch und antipruriginös $[10,17]$.
Mit zunehmender Lufttemperatur nimmt bei gleichbleibender relativer Luftfeuchtigkeit automatisch die absolute Luftfeuchtigkeit, der so genannte Luftdampfdruck, zu, was direkt proportional zu einer Erhöhung des Wassergehaltes in der Epidermis führt $[25,26]$. Dieses ist beim atopischen Ekzem von besonderer Bedeutung, da Wasserbindungskapazität und Wassergehalt des Stratum corneum bei Neurodermitikern oft erniedrigt sind [27], was zu Irritation [28] und Pruritus [21] führt. Eine höhere Hautfeuchtigkeit dagegen wirkt pruritusmindernd. Dieser Zusammenhang bestätigte sich in der hochsignifikanten inversen Korrelation zwischen Luftdampfdruck und Pruritus.

Die pruritusmindernden Effekte höherer Sonnenscheindauer erklären sich einerseits durch die luftaustrocknende und temperaturerhöhende Wirkung der Sonnenstrahlung $[19,20]$ andererseits durch die therapeutische immunologische Wirkung der UV-Strahlung selbst $[17,20]$. Da der Pruritus bemerkenswerterweise weniger von der Sonnenscheindauer als von der Lufttemperatur abhing, lässt sich vermuten, dass die UV-Strahlung beim atopischen Ekzem nicht von primärer therapeutischer Bedeutung ist. Die verschiedenen Bewölkungsgrade hatten dementsprechend keine nennenswerte Relevanz, ganz abgesehen davon, dass an einem mäßig wolkigen Tag durchaus mehr UV-Strahlung als an einem klaren Tag einstrahlen kann [20].

Subjektive Wetterempfindungsqualitäten sind individuell unterschiedlich und Ausdruck autonomer Anpassungsvorgänge des menschlichen Organismus an klimatische Gegebenheiten $[20,23]$. Bei Behaglichkeit empfindet der Mensch die Umgebungsluftbedingungen als indifferent und die vegetative Regulation ist im vollständigen Gleichgewicht. Wenn dagegen die meteorologischen Umgebungsbedingungen nicht mittels autonomer Adaptionsmechanismen vollständig kompensiert werden können, registriert der Mensch die Gegenregulationen, er empfindet das Klima als different, z.B. kalt, windig, feucht etc.; es liegt Unbehagen, meteorotropisch als Diskomfort bezeichnet, vor $[20,23,24]$.

Feuchteempfinden bei über $50 \%$ der Patienten trat bei erhöhter relativer Luftfeuchtigkeit von durchschnittlich $86 \%$ in Verbindung mit niedrigen Lufttemperaturen von durchschnittlich $2,9^{\circ} \mathrm{C}$ auf. Das Gefühl von feuchter Kälte entsteht im Allgemeinen bei Lufttemperaturen zwischen $12^{\circ} \mathrm{C}$ und $15^{\circ} \mathrm{C}$ und hoher relativer Luftfeuchtigkeit [19]. Die Patienten des untersuchten Kollektivs empfanden „Feuchtigkeit“ jedoch bei niedrigeren Lufttemperaturen von durchschnittlich $2,9^{\circ} \mathrm{C}$, bei welchen normalerweise das Gefühl von feuchter Kälte nicht aufkommen kann, weil bei Lufttemperaturen $<12{ }^{\circ} \mathrm{C}$ selbst bei $100 \%$ iger Luftfeuchtigkeit der Dampfdruck nie die Schwülegrenze von 18,8 hPa erreicht $[19,20,23]$. Die Patienten haben somit eine erhöhte Sensibilität für Luftfeuchtigkeit: Luftfeuchtigkeit wird bereits in Bereichen, die der Hautgesunde unbemerkt kompensiert, subjektiv wahrgenommen, weil hierbei vermutlich bereits der für den Wärmeübergang an der Haut erforderliche TEWL behindert wird. Auch für Lufttrockenheit war die Empfindlichkeit gesteigert, da bei einer relativen Luftfeuchtigkeit von $67 \%$ (bei durchschnittlicher Lufttemperatur von $5,1^{\circ} \mathrm{C}$ und Dampfdruck von $6,14 \mathrm{hPa}$ ) im Durchschnitt 50\% der Patienten das Wetter bereits als trocken empfanden. Eine leicht bekleidete Normalperson dagegen empfindet bei einer relativen Luftfeuchtigkeit von $40-60 \%$ und einer Lufttemperatur von $18-20^{\circ} \mathrm{C}$ normalerweise Behaglichkeit [19]. 
Dass Neurodermitiker auf erniedrigte Luftfeuchtigkeitsgrade früher als Hautgesunde mit Irritation reagieren, konnte in einer Untersuchung in der Klimakammer gezeigt werden [29].

Die erhöhte subjektive Empfindlichkeit, speziell für Luftfeuchtigkeit und Lufttrockenheit, korrelierte in unserer Untersuchung jedoch nicht nennenswert mit verstärktem Pruritus, d. h., der meteoreologische Diskomfort setzt bereits unter Bedingungen ein, unter denen noch keine klinischen Symptome entstehen. Somit erweist sich das subjektive Wetterempfinden bei Atopikern als hochsensibler frühzeitiger Indikator für ungünstige thermischhygrische Bedingungen.

Schlussfolgernd kann nach dieser Untersuchung angenommen werden, dass sich ein für Neurodermitis günstiges Klima offensichtlich außer durch mittlere, nicht zu intensive UV-Strahlung vor allem dadurch auszeichnet, dass eine ständige Wärmeabgabe an der Hautoberfläche möglich ist und gleichzeitig die Haut nicht zu sehr austrocknet. Günstig sind mittlere Lufttemperaturen, die luftdurchlässige Kleidung, einen guten Wärmegradienten an der Hautoberfläche und einen ausreichenden Luftdampfdruck gewährleisten, sowie eine für Außenbedingungen eher niedrige relative Luftfeuchtigkeit um $70 \%$ und angemessene Luftbewegung. Es scheinen sich für Neurodermitiker in diesem Zusammenhang „optimale Bereiche“ herausarbeiten zu lassen, extreme Bedingungen sind in jedem Fall ungünstig. Neurodermitiker haben konstitutionell einen nach oben und unten eingeschränkten thermisch-hygrischen Toleranzbereich, was sich mit erhöhter Sensibilität im subjektiven Komfortempfinden manifestiert.

Inwieweit die Ergebnisse dieser Untersuchung sich unter anderen Klimaverhältnissen bestätigen bzw. erweitern lassen und wie spezifisch sie für Neurodermitiker sind, müssen vergleichende Studien in anderen, auch erfahrungsgemäß ungünstigen Re-

Die Daten geben jedoch bereits jetzt Anlass, die Relevanz des umgebenden Klimas für den Verlauf des atopischen Ekzems zu berücksichtigen und entsprechend praktisch umzusetzen, speziell bei Fragen zu Wohnortwahl, Arbeitsplatz, Bekleidungsgewohnheiten, Freizeit- und Urlaubsverhalten sowie therapeutischen und insbesondere klimatherapeutischen Maßnahmen.

\section{Danksagung}

Herrn Dr. Claus Fröhlich, PMOD/WRC Davos, und der SMA Zürich danken wir für die Überlassung der meteorologischen Daten.

\section{Literatur}

${ }^{1}$ Borelli S, Schnyder UW. Neurodermitis constitutionalis sive atopica. In: Jadassohn J (Hrsg). Handbuch der Haut- und Geschlechtskrankheiten - Ergänzungswerk Vol II/1. Berlin, Göttingen, Heidelberg: Springer, 1962

2 Imai S, Takeuchi S, Mashiko T. Jahreszeitliche Änderungen im Verlauf des atopischen Ekzems. Hautarzt 1987; 38: 599-602

${ }^{3}$ Spitzer R. Geographische Verteilung der Hautkrankheiten. In: Marchionini A (Hrsg). Beiträge zum Handbuch der Haut- und Geschlechtskrankheiten - Ergänzungswerk Vol VIII. Berlin: Springer, 1967
${ }^{4}$ Hanifin JM. Atopic dermatitis. J Allergy Clin Immunol 1984; 73 : $211-222$

${ }^{5}$ Rajka G. Atopic dermatitis. Correlation with environmental factors. Int J Dermatol 1986; 25: 301 - 304

${ }^{6}$ Pürschel W. Klimatherapie der Neurodermitis atopica an der Nordsee. Allergologie 1982; 5: 64-69

${ }^{7}$ Steiger T, Borelli S. Significance of climatic factors in the treatment of atopic eczema. In: Ruzicka T, Ring J, Przybilla B (Hrsg). Handbook of atopic eczema. Berlin, Heidelberg: Springer, 1991

${ }^{8}$ Vocks E, Borelli S, Rakoski J. Klimatherapie bei Neurodermitis. Allergologie 1994; 17: 208 - 213

${ }^{9}$ Cooper KD. Atopic Dermatitis: Recent trends in pathogenesis and therapy. J Invest Dermatol 1994; 102: 128-137

${ }^{10}$ Ryan TJ. Vascular reactivity in atopic eczema. In: Ruzicka T, Ring J, Przybilla B (Hrsg). Handbook of atopic eczema. Berlin, Heidelberg: Springer, 1991

${ }^{11}$ Heyer G, Berg P, Hornstein OP. Abnormal cutaneous vasoreactions in atopics during and after acute eczema. Hautarzt 1995; 46: 22 - 27

12 Rajka G. Non-immunological factors in the aetiology of atopic dermatitis. In: Rajka G (Hrsg). Atopic dermatitis. London, Philadelphia, Toronto: WB Saunders Company Ltd, 1975

${ }^{13}$ Fartasch M, Diepgen T. The barrier function in atopic dry skin. Disturbance of membrane coating granule exocytosis and formation of epidermal lipids? Acta Derm Venereol (Stockh) 1992; 176: 26-31

14 Tupker RA, Pinnagoda J, Coenraads PJ, Nater JP. Susceptibility to irritants: Role of barrier function, skin dryness and history of atopic dermatitis. Br J Dermatol 1990; 123: 199-205

${ }^{15}$ Leung DYM. Atopic dermatitis: The skin as a window into the pathogenesis of chronic allergic diseases. J Allergy Clin Immunol 1995; 96: $302-319$

${ }^{16}$ Rothe MJ, Grant-Kels JM. Atopic dermatitis: an update. J Am Acad Dermatol 1996; 35: 1 - 13

${ }^{17}$ Ring J, Abeck D, Brockow K. The therapeutic concept of „patient management" in atopic eczema. Allergy 1996; 51: $206-215$

18 Przybilla B, Ring J, Kejzlar-Lisy G. Behandlung des atopischen Ekzems mit UVAPUR - klinische und immunologische Ergebnisse. Akt Dermatol 1988; 14: 326-329

${ }^{19}$ Daubert K, Aichinger F. Wetter - Klima - Haut. In: Gottron HA, Schönfeld W (Hrsg). Dermatologie und Venerologie Vol I/2. Stuttgart: Thieme, 1958

${ }^{20}$ Flach E. Human bioclimatology. In: Landsberg HE (Hrsg). World survey of climatology, Vol 3: General climatology. Amsterdam: Elsevier Scientific Publishing Company, 1981

${ }^{21}$ Hägermark Ö. The pathophysiology of itch. In: Ruzicka T, Ring J, Przybilla B (Hrsg). Handbook of atopic eczema. Berlin, Heidelberg: Springer, 1991

${ }^{22}$ Wahlgren CF. Itch and atopic dermatitis: clinical and experimental studies. Habilitationsschrift: Department of Dermatology Stockholm Kopolinska Hospital, 1991

${ }^{23}$ Wiedemann E. Klimatherapie. In: Wiedemann E (Hrsg). Physikalische Therapie, Grundlagen - Methoden - Anwendung. Berlin, New York: de Gruyter, 1987

24 Jendritzky G. Wirkungen von Wetter und Klima auf die Gesundheit des Menschen. In: Wichmann HE, Schlipköter HW, Fülgraff G (Hrsg). Handbuch der Umweltmedizin. Landsberg/Lech: ecomed, 1992

${ }^{25}$ Pinnagoda J, Tupker RA, Agner T, Serup J. Guidelines for transepidermal water loss (TEWL) measurements. Contact dermatitis 1990; 22: $164-178$

${ }^{26}$ Spencer TS, Linamen EC, Akerds WA, Jones HE. Temperature dependence of water content of stratum corneum. Br J Dermatol 1975; 93: $159-164$

${ }^{27}$ Werner Y. The water content of the stratum corneum in patients with atopic dermatitis. Measurement with the corneometer CM 420. Acta Derm Venereol (Stockh) 1986; 66: 281 - 284

${ }^{28}$ Nickoloff BJ, Naidu Y. Perturbation of epidermal barrier function correlates with initiation of cytokine caskade in human skin. J Am Acad Dermatol 1994; 30: 535-546

${ }^{29}$ Eberlein-König B, Spiegl A, Przybilla B. Change of skin roughness due to lowering air humidity in a climate chamber. Acta Derm Venereol (Stockh) 1996; 76: 447-449 